Check for updates

Cite this: RSC Adv., 2017, 7, 47966

\title{
Near-infrared emitting CdTeSe alloyed quantum dots: Raman scattering, photoluminescence and single-emitter optical properties $\uparrow$
}

Le Xuan Hung, ${ }^{\text {ab }}$ Pascal D. Bassène, ${ }^{\text {cd }}$ Pham Nam Thang, ${ }^{\text {be }}$ Nguyễn Thu Loan, ${ }^{\text {ce }}$ Willy Daney de Marcillac, ${ }^{c}$ Amit Raj Dhawan, ${ }^{\text {fc }}$ Fu Feng, ${ }^{\mathrm{C}}$ Juan U. Esparza-Villa, ${ }^{\mathrm{c}}$ Nguyen Thi Thuc Hien, ${ }^{a}$ Nguyen Quang Liem, ${ }^{e}$ Laurent Coolen ${ }^{c}$ and Pham Thu Nga*abe

The synthesis of ternary core/shell zinc-blende CdTeSe/ZnSe quantum dots with optimal synthesis parameters is analyzed. For the synthesis of the ternary-alloy CdTeSe core, a temperature around $260^{\circ} \mathrm{C}$ is shown to allow a good reaction of both the Te and Se reagents, while for lower temperatures mostly$\mathrm{CdTe}$ quantum dots are synthesized. At this temperature, by changing the ratio of the $\mathrm{Te}$ and $\mathrm{Se}$ reagents, the composition can be tuned from Te-rich to Se-rich while keeping a constant quantum dot size. The photoluminescence wavelength expands up to $860 \mathrm{~nm}$ for the core CdTeSe and $900 \mathrm{~nm}$ for the CdTeSe/ZnSe samples, a near-infrared bandgap with potential applications in photovoltaics. The use of Raman spectroscopy is proved especially useful to analyze the composition of the alloy quantum dots, with the presence of two longitudinal-optical phonon lines corresponding respectively to CdTe and CdSe vibrations. For single core/shell CdTeSe/ZnSe quantum dots, perfect single-photon emission is demonstrated with good stability and low blinking, and a long decay time of 110 ns suggesting high luminescence quantum yield.

rsc.li/rsc-advances

\section{Introduction}

Colloidal quantum dots (QDs) are the subject of intense research programs and innovations to be used in different fields such as solar cells, photocatalysis, light-emitting diodes or biosensors. ${ }^{1-6}$ Numerous studies on types of QDs that can absorb strongly in the near-infrared (NIR) range (700-900 nm) are currently being conducted, since these QDs can be used as an absorber of solar radiation. ${ }^{7-11}$ Alloy QDs have attracted great attention due to the enhanced chemical stability resulting from a hardened lattice structure, higher crystallinity, decreased inter diffusion processes and higher photoluminescence

\footnotetext{
${ }^{a}$ Institute of Research and Development, Duy Tan University, Danang, Vietnam. E-mail: ngalamvn@gmail.com

${ }^{b}$ Graduate University of Science and Technology, Vietnam Academy of Science and Technology, 18 Hoang Quoc Viet Road, Cau giay Dist., Hanoi, Vietnam

'Sorbonne Universités, UPMC Univ Paris 06, UMR 7588, Institut de NanoSciences de Paris (INSP), Paris F-75005, France

${ }^{d}$ Groupe de Laboratoires Physique des Solides et Sciences de Matériaux (GPLSSM), Université Cheikh Anta Diop de Dakar (UCAD), Senegal

${ }^{e}$ Institute of Materials Science, Vietnam Academy of Science and Technology, 18 Hoang Quoc Viet Road, Cau Giay Dist., Hanoi, Vietnam

${ }^{f}$ Institute of Fundamental and Frontier Sciences, University of Electronic Science and Technology of China, Chengdu 610054, People's Republic of China

† Electronic supplementary information (ESI) available. See DOI: 10.1039/c7ra06500k
}

quantum yields. ${ }^{12}$ The optoelectronic properties of alloyed QDs can be tuned by their composition without changing the particle size. ${ }^{13}$ Besides, ternary alloy cadmium selenide telluride $\left(\mathrm{CdTe}_{x} \mathrm{Se}_{1-x}\right)$ QDs can absorb at lower energies when compared with their parent binary CdSe and CdTe QDs, so that, alloyed ternary QDs have many significant advantages compared to their parent binary semiconductors. The possibility to tune both their size and composition, possibly including composition gradients in the material, offers additional degrees of freedom. In particular, due to the optical bowing effect, the absorption and emission wavelengths of the ternary alloy can be redshifted further than for its parent binary semiconductors. For instance, for CdTeSe QDs, expansion of the absorption range up to $\sim 900 \mathrm{~nm}$ has been reported. ${ }^{3,4} \mathrm{CdTe}_{x} \mathrm{Se}_{1-x}$ alloyed QDs with water-soluble mercaptopropionic acid (MPA) ligand were investigated in 2013 by Pan et al. ${ }^{\mathbf{1 1 , 1 3}}$ applied in a liquid junction QD sensitized solar cell. In $^{11} \mathrm{CdTe}_{x} \mathrm{Se}_{1-x}$ alloyed QDs were used for the first time in solid state organic solar cells.

Many fabrication methods have been studied and improved to synthesize CdTeSe QDs with core/shell structure, ${ }^{14-19}$ and different kinds of shells such as ZnSe or ZnTe have been reported lately. ${ }^{20}$ Due to the reactivity difference between Te and Se, a CdTe-rich core and CdSe-rich shell have been formed in the CdTeSe alloy with the formation of a gradient type II. ${ }^{21}$ This study was carried out with the aim to understand more thoroughly the conditions to fabricate CdTeSe QDs that only 
crystallize in the zinc-blende single crystalline phase, by using a simple modified fabrication method described in. ${ }^{\mathbf{1 3 , 1 7 , 2 2 - 2 5}}$ CdTeSe/CdS had been used in quantum-dot-sensitized solar cells with power conversion efficiency achieving over $9 \%{ }^{7}$ Recently, Poplawsky et al. showed the role of the CdTeSe layer as a NIR photoactive layer in CdTe-based solar cells ${ }^{\mathbf{2 6}}$ and for using them in quantum-dot-sensitized solar cells (QDSC), we have conducted detailed studies on this material.

In this paper, we present our studies on alloyed CdTeSe QDs with NIR absorption and emission, with the aim of fabricating single-phase zb-CdTeSe QDs with a process in a solution at a suitable temperature $\left(<300{ }^{\circ} \mathrm{C}\right)$. A systematic study is conducted on the effects of temperature and reagent ratios on structural and optical properties. A temperature of $260{ }^{\circ} \mathrm{C}$ is found optimal for incorporation of both Te and Se into the QDs. Raman spectra, X-ray analysis and optical spectroscopy consistently show that the composition of the alloyed QDs can be tuned through the Te and Se reagents concentration. The growth of a ZnSe shell is also described and microphotoluminescence study at the single quantum dot level has shown that the QDs emit single photons, with limited PL blinking.

\section{Experimental section}

\section{Synthesis method of CdTeSe and CdTeSe/ZnSe QDs}

The fabrication method of CdTeSe ternary QDs has been described in our recent publication ${ }^{20}$ and reported in. ${ }^{27-29} \mathrm{We}$ used the following reagents (from Aldrich) - cadmium acetate dihydrate $\left(\mathrm{Cd}(\mathrm{Ac})_{2} \cdot 2 \mathrm{H}_{2} \mathrm{O}, 99.9 \%\right)$ as a source of $\mathrm{Cd}$, zinc acetate $\left(\mathrm{Zn}(\mathrm{Ac})_{2}, 99.9 \%\right)$ as a source of $\mathrm{Zn}$, elemental selenium powder (Se, 99.99\%) as a source of Se, elemental tellurium powder (Te, 99.99\%) as a source of Te, oleic acid (OA, 90\%) and oleylamine (OLA, 90\%) as surface ligands, 1-octadecene (ODE, 90\%) and trioctylphosphine (TOP, 90\%) as the reaction medium. All chemicals were used without further purification.

We first fabricated the precursor solutions by taking $1.33 \mathrm{~g}$ ( $5 \mathrm{mmol}$ ) of cadmium acetate dihydrate and dissolved it in $1.59 \mathrm{ml}(5 \mathrm{mmol})$ of $\mathrm{OA}$ and $75 \mathrm{ml}$ of ODE. $5 \mathrm{ml}$ of OLA $(15 \mathrm{mmol})$ are added to the mixture of OA and ODE to prepare Cd precursor for QD samples of the first series, following ref. 15 where the growth was at $230{ }^{\circ} \mathrm{C}$. However for the second and third series, grown at higher temperatures $\left(260{ }^{\circ} \mathrm{C}\right)$, OLA is not added as at high temperatures it would cause the QDs to quickly change to brown and immediately deposit (see discussion ESI $\dagger$ ). The TOP-Se and TOP-Te precursors were fabricated by dissolving respectively $31.6 \mathrm{mg}(0.4 \mathrm{mmol})$ of powder Se in $0.4 \mathrm{ml}$ of TOP and $76.6 \mathrm{mg}(0.6 \mathrm{mmol})$ of Te metal in $1 \mathrm{ml}$ of TOP, at 80$100{ }^{\circ} \mathrm{C}$, in $\mathrm{N}_{2}$ gas. Then, all amount of these precursors were brought into a reaction flask. We quickly injected the mixed precursors TOP-Se and TOP-Te into a three-neck flask containing the Cd precursor solution at $120{ }^{\circ} \mathrm{C}$ for $1 \mathrm{~h}$, in $\mathrm{N}_{2}$ gas.

For a first series of CdTeSe QD samples, we used a constant Cd: Te:Se molar fraction 5:0.6:0.4 with the mass as described above, but performed the growth reaction at different temperatures: $180{ }^{\circ} \mathrm{C}, 200{ }^{\circ} \mathrm{C}, 220^{\circ} \mathrm{C}, 240{ }^{\circ} \mathrm{C}, 260{ }^{\circ} \mathrm{C}$ or $280{ }^{\circ} \mathrm{C}$. We kept this temperature stable for a period of $10 \mathrm{~min}$, while vigorously stirring the reacting solution, to create nanoparticle seeds and grow them. Then we allowed the solution to cool slowly while stirring with a magnetic stirrer. Finally, we obtained $\sim 85.5 \mathrm{ml}$ solution containing colloidal quantum dots, corresponding to $1 \mathrm{mmol}$ quantum dots.

In a second series of syntheses of CdTeSe QDs, we used different Te fractions $(\mathrm{Te} /(\mathrm{Te}+\mathrm{Se})$ ratio $), x=0,0.2,0.4,0.5,0.6$, 0.8 and 1 , with the precursors fabricated in a similar way as above - note that $x$ is the fraction of Te reagent, not the fraction of Te inside the CdTeSe QDs. The nanocrystals growth temperature was then $260{ }^{\circ} \mathrm{C}$ for $10 \mathrm{~min}$. We obtained $79.6 \mathrm{ml}$ solution containing colloidal quantum dots, corresponding to $1 \mathrm{mmol}$ quantum dots.

A third series of samples of CdTeSe/ZnSe core/shell QDs was synthesized from CdTeSe cores $\left(T=260{ }^{\circ} \mathrm{C}, x=0.5\right)$ with different ZnSe shell thicknesses. The process of preparing the precursors for $\mathrm{Zn}$ and Se is completely identical to that of the initial Cd precursor and Se-TOP. The masses of $\mathrm{Zn}$ and Se were calculated for 1 monolayer (ML), $2 \mathrm{ML}, 4 \mathrm{ML}$ and $6 \mathrm{ML}$ of ZnSe. An ML thickness is based on the lattice constant $a$ of $\mathrm{ZnSe}$ crystals, depending on the type of shell. The molar ratio of $\mathrm{Zn}: \mathrm{Se}$ was $1: 1$. After cleaning out the ligands and unreacted residue substances, we took $46.4 \mathrm{ml}$ of the CdTeSe core QD solution ( $\sim 1.6 \mathrm{mmol}$ ) and poured it in to a three-necked flask, and quickly raised the temperature to $230{ }^{\circ} \mathrm{C}$. At this temperature, we quickly injected $2.8 \mathrm{ml}$ of the $\mathrm{Zn}$ precursor solution (corresponding to one monolayer of $\mathrm{Zn}$ ions) and stirred vigorously for $15 \mathrm{~min}$. Then, we quickly injected $1.3 \mathrm{ml}$ of TOPSe and stirred vigorously for 15 min to grow the shell. Next, we took out $25 \mathrm{ml}$ of the solution containing QDs, which constituted a first CdTeSe/ZnSe sample with $1 \mathrm{ML}$ nominal shell thickness. With the remaining volume, we continued to quickly inject $1.4 \mathrm{ml}$ of $\mathrm{Zn}$ precursor, stirred vigorously for $10 \mathrm{~min}$, then injected TOP-Se $(0.7 \mathrm{ml})$, stirred vigorously to grow the ZnSe particles' shell for $15 \mathrm{~min}$. We obtained the CdTeSe/ZnSe QD sample of nominal shell thickness $2 \mathrm{ML}$. We performed the same operations when coating ZnSe for CdTeSe QD cores to form CdTeSe/ZnSe with higher thickness such as 4 and 6 ML.

All alloyed ternary quantum dots were purified by several rounds of precipitation and centrifugation and were stored at room temperature for later characterization and use.

\section{Characterization of alloyed CdTeSe ternary quantum dots}

The size of the CdTeSe QDs was determined by transmission electron microscopy (TEM) with a JEOL Jem 1010 microscope operating at $100 \mathrm{kV}$. Powder X-ray diffraction (XRD, Siemens D5005) was used to confirm the wurtzite (w) or zinc-blende (zb) crystalline structure.

The ultraviolet-visible (UV-Vis) absorption spectra of the QDs in toluene were measured within the wavelength range of 200$1000 \mathrm{~nm}$ using a Shimadzu (UV-1800) UV-Vis spectrophotometer. All UV-Vis measurements were performed at $25{ }^{\circ} \mathrm{C}$ and automatically corrected for the solvent medium.

The PL spectra measurement was carried out on a Fluorolog322 system (Jobin-Yvon) by using Xenon $450 \mathrm{~W}$ light with excitation wavelength selected at $532 \mathrm{~nm}$. The detector is 
a photomultiplier, measuring range from 250-1000 nm. All PL spectra were measured under the same conditions. The solution concentrations were adjusted in order to all present an optical density of 0.03 at $532 \mathrm{~nm}$ so that the emission intensities of the different samples can be compared (although affected by the measurement system spectral response).

For the PL decays ensemble measurements, a pulsed nitrogen laser $(337 \mathrm{~nm}$, pulse width $0.6 \mathrm{~ns}$, repetition rate of 10 $\mathrm{Hz}$ ) was used as the excitation source. The PL from the samples was collected by an optical fiber on the same side as the excitation light, then was analyzed by a Jobin-Yvon Spectrometer HR460 and the PL decays were measured with a PM Hamamatsu R5600U and a Tektronix TDS 784A scope with the time resolution of $1 \mathrm{~ns}$.

For individual QD measurements, the QDs were spin-coated on a glass coverslip and then covered them by a $50 \mathrm{~nm}$ layer of PMMA to protect them from the oxidation. The sample was imaged with a microscope and $\mathrm{a} \times 60$ oil-immersion objective with 0.7 numerical aperture. A single QD was excited by a $450 \mathrm{~nm}$ pulsed diode laser. Its emission was selected by a pinhole located in the objective image plane, and detected by two avalanche photodiodes in Hanbury-Brown and Twiss configuration, connected to a Picoharp acquisition card. The overall setup provided a 400 ps resolution.

The QD samples were analyzed by Micro Raman spectroscopy (XploRA-Horiba) using $532 \mathrm{~nm}$ (25 mW) excitation line from a diode-pumped, solid state laser to analyze the vibration bonds and their Raman frequencies. The laser power was 100 $\mathrm{mW} .10 \times$ objectives were used to focus the excitation laser light on the right spot of the investigated samples. The spot size of the laser beam was $1 \mu \mathrm{m}$. The spectral resolution was $2 \mathrm{~cm}^{-1}$. The acquisition time ranged from $30 \mathrm{~s}$ to $120 \mathrm{~s}$, but normally was $30 \mathrm{~s}$. The system uses a Charge Coupled Device (CCD) receiver with four gratings 600, 1200, 1800 and $2400 \mathrm{~g} \mathrm{~mm}^{-1}$, measuring from 100 to $4000 \mathrm{~cm}^{-1}$. For Raman and PL measurements at low temperature (liquid nitrogen temperature 77 to $293 \mathrm{~K}$ ) we combined the Raman XploRA Plus system with the THMS 600 Linkam temperature control system. The whole THMS6 00 system is controlled by the Lynksys-32 software with temperature adjustment of $0.1 \mathrm{~K}$.

With powder X-ray diffraction (XRD) and Raman measurements, the CdTeSe QD samples were used in solid form. These samples were purified by washing thrice with isopropanol. The sample that was used to measure TEM, absorption and fluorescence spectra was in solution in toluene, after being purified of ligands and remaining excess substances after QD fabrication.

\section{Results and discussion}

\section{Effect of temperature on the growth of CdTeSe ternary quantum dots}

We first consider the series of CdTeSe QDs fabricated with constant Te : Se ratio (0.6:0.4), but different growth temperatures from $180{ }^{\circ} \mathrm{C}$ to $280{ }^{\circ} \mathrm{C}$.

Optical properties. Fig. 1 shows the emission spectra of the different CdTeSe QD samples. The maximum peak of the emission band shifts from $\sim 716 \mathrm{~nm}$ for the sample grown at

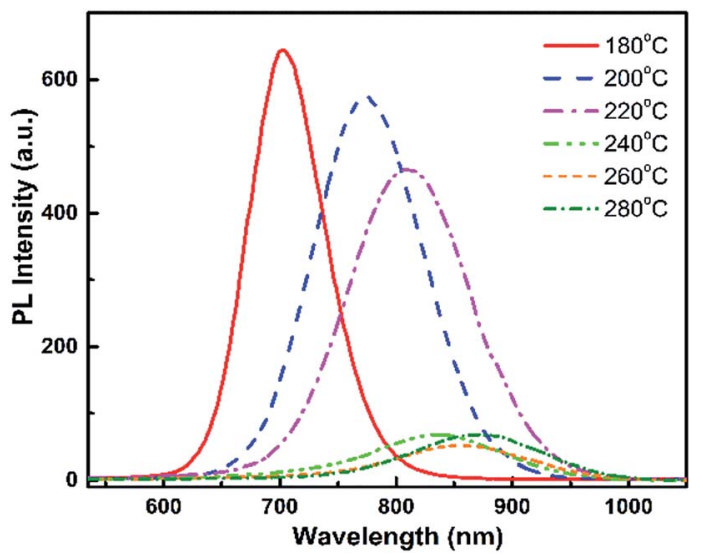

Fig. 1 The emission spectra of QD samples grown at different temperatures from $180^{\circ} \mathrm{C}$ to $280^{\circ} \mathrm{C}$.

$180{ }^{\circ} \mathrm{C}$ to $860 \mathrm{~nm}$ for QD samples grown at $280{ }^{\circ} \mathrm{C}$. So, there is a shift in the emission spectrum of approximately $144 \mathrm{~nm}$ towards longer wavelengths when the nanocrystal growth temperature is increased. The reason behind this shift might be an increase in the nanocrystals' size or a change in the QDs' composition depending on the growth temperature, as will be further analyzed below. The PL intensity is shown to decrease sharply above $220{ }^{\circ} \mathrm{C}$ growth temperature.

The absorption spectra of this sample series show that there is a change in absorption intensity and edge when nanocrystal growth temperature is increased (Fig. 2). The absorption edge is shifted towards longer wavelengths for higher growth temperature, in agreement with the PL spectra. Thus, the absorption capability of QDs fabricated at $240{ }^{\circ} \mathrm{C}$ to $260{ }^{\circ} \mathrm{C}$ increases notably in the NIR region. The absorption and emission wavelengths are in the same range as values previously reported by Bailey et al., ${ }^{4}$ but redshifted with respect to other samples described in. ${ }^{\mathbf{1 3 , 1 5 , 2 2}}$ The photoluminescence decay curves (see ESI, Fig. SI-1†) present two characteristic times, one of 10-40 ns corresponding to the exciton radiative recombination, and the other of the order of a few nanoseconds which can be attributed to the presence of non-radiative decay channels.

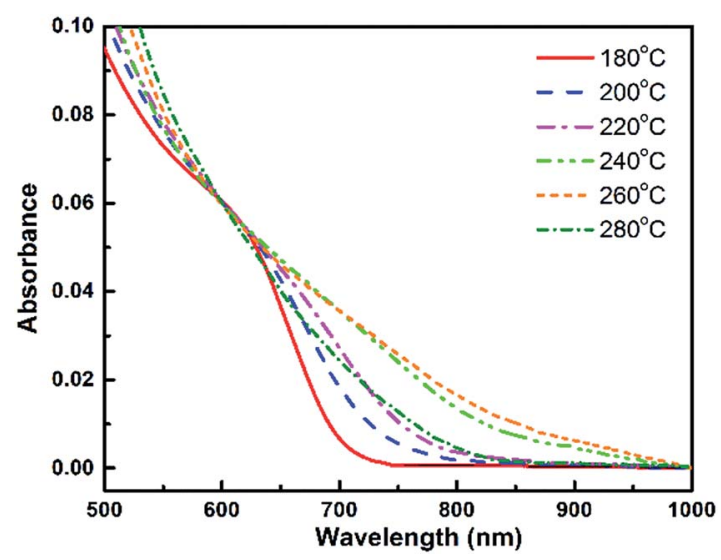

Fig. 2 The absorption spectra of QDs grown at different temperatures from $180^{\circ} \mathrm{C}$ to $280{ }^{\circ} \mathrm{C}$. 
In order to analyze the reasons for the spectral shift between these different samples, we have recorded the micro-Raman spectra, XRD patterns and TEM images of all samples to obtain more information on QDs composition, shape and size.

Raman scattering spectra. The Raman scattering spectrum of II-VI ternary alloyed QDs is known to display different vibrational characteristics, depending on the atomic masses of the alloy constituents. ${ }^{30-32}$ The Raman and IR spectra of $\mathrm{CdTe}_{x} \mathrm{Se}_{1-x}$ crystals suggested the possibility of two-phononmode behaviour. ${ }^{33-35}$ An unexpected phonon feature in $\mathrm{CdTe}_{1-x} \mathrm{Se}_{x}$ was interpreted ${ }^{33}$ as a tendency of the alloy to form small clusters either CdTe-rich or CdSe-rich. This was believed to act on the optical phonon properties as they depend strongly on the Cd-Se, Cd-Te nearest-neighbor bond lengths/force constants. ${ }^{31}$ so that eventually optical phonon frequencies close to the bulk CdTe or CdSe frequencies are found..$^{32}$

Fig. 3 displays the Raman spectra of the CdTeSe QDs samples series fabricated at different temperatures from $180{ }^{\circ} \mathrm{C}$ to $280^{\circ} \mathrm{C}$. In the frequency range of 125 and $225 \mathrm{~cm}^{-1}$, the Raman spectra have revealed CdTe-like and CdSe-like longitudinal optical (LO) phonons, labeled $\mathrm{LO}_{1}$ and $\mathrm{LO}_{2}$ respectively.

For the growth at $180{ }^{\circ} \mathrm{C}$, only one vibration band appears at $159 \mathrm{~cm}^{-1}$ - characteristic of the CdTe-like LO mode, ${ }^{27,30,36}$ and a shoulder with lower intensity at $188 \mathrm{~cm}^{-1}$ (CdSe-like LO mode). ${ }^{36,37}$ As the growth temperature is increased, the second peak at $188 \mathrm{~cm}^{-1}$ now appears distinctly. It increases gradually and becomes equal to the peak at $159 \mathrm{~cm}^{-1}$ for $260{ }^{\circ} \mathrm{C}$, and quite higher at $280^{\circ} \mathrm{C}$. We can conclude that the QDs synthesized at lower temperatures are CdTe-rich, due to the stronger Te reactivity, and the incorporation of Se into the QDs increases with temperature. As it was shown in ref. 38 for CdSeS quantum dots that the ratio between Raman peaks is close to the ratio between concentrations we can estimate that similar amounts of Te and Se are included in the QDs around $260{ }^{\circ} \mathrm{C}$. Given that similar amounts of Te and Se were introduced, we interpret this as a sign that, at $260{ }^{\circ} \mathrm{C}$, the Te and Se are incorporated into the QDs with similar efficiencies. This temperature is thus optimal in order to achieve various compositions of the CdTeSe alloy by

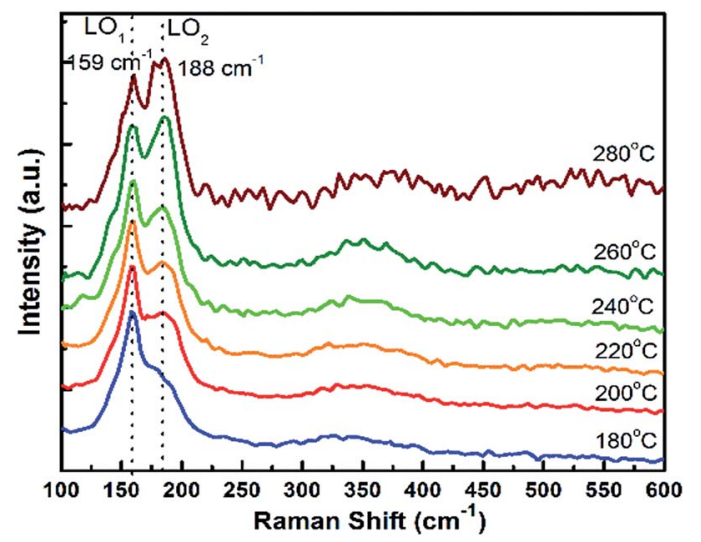

Fig. 3 Raman spectra of CdTeSe QDs prepared at different temperatures, from $180^{\circ} \mathrm{C}$ to $280^{\circ} \mathrm{C}$. choosing different ratios of the Se and Te reagents, as will be demonstrated in the next section.

$\mathrm{X}$ rays diffraction. The crystalline phases of these different CdTeSe QD samples have been analyzed by XRD (see ESI, Fig. SI$4 \dagger)$. The major diffraction peaks of the (111), (220) and (311) planes were observed, corresponding to a typical cubic zinc blend (zb) structure. These peaks are located between the corresponding tabulated lines of bulk $\mathrm{zb}$ CdSe and CdTe. Therefore, we can conclude that the CdTeSe fabricated are all crystalline single phase zb-CdTeSe. A slight shift of these peaks towards higher angles was observed with increased growth temperature, in agreement with the increase of Se concentration demonstrated by Raman scattering. However XRD appeared a much less powerful tool than Raman spectroscopy for the analysis of CdTeSe alloying effects.

TEM images. From the obtained TEM images of samples CdTeSe QDs prepared at temperature from $180^{\circ} \mathrm{C}$ to $280^{\circ} \mathrm{C}$ (see ESI, Fig. SI- $\left.{ }^{\dagger}\right)$, CdTeSe QDs fabricated at $180-220{ }^{\circ} \mathrm{C}$ have a rather spherical shape. On higher QD growth temperatures, their shape seems to become more elongated. At $280{ }^{\circ} \mathrm{C}$, this looks more obvious and there are more elongated particles than spherical ones. The average sizes of the six QD samples (in the longer dimension, estimated over 80-90 particles), are respectively 6.2, 6.8, 7.3, 8.2, 8.7 and $9.2 \mathrm{~nm}$ for the CdTeSe QDs prepared at $180^{\circ}, 200^{\circ}, 220^{\circ}, 240^{\circ}, 260^{\circ}$ and $280^{\circ} \mathrm{C}$. The QD size is increased with the growth temperature.

Therefore, an increase in QD growth temperature increases their size as well as their Se concentration. The size increase leads to reduced quantum confinement and thus can explain the observed redshifted luminescence at higher growth temperature. The higher Se concentration also contributes to this redshift (note that, due to the optical bowing effect, adding a small amount of Se to CdTe leads to a luminescence redshift although CdSe has a higher bandgap energy than CdTe). As explained previously, the growth temperature of $260{ }^{\circ} \mathrm{C}$ will be chosen as it allows the best incorporation of both Te and Se into the alloy QDs. The PL efficiency was found to decrease above $240{ }^{\circ} \mathrm{C}$, however further characterizations (see discussion in ESI, Fig. SI-5 and SI-6†) showed that significantly higher PL efficiencies could be obtained, especially at high temperatures, without changing the resulting Se : Te composition and its dependence of temperature, by removing the OLA ligand from the precursor solution. We will use this modified protocol in the following.

\section{Effect of the change of the Te/Se precursors ratio}

We now consider the second CdTeSe QD samples series fabricated at $260{ }^{\circ} \mathrm{C}$ for $10 \mathrm{~min}$, with only the $\mathrm{Te} /(\mathrm{Te}+\mathrm{Se})$ precursors ratio $(x)$ changed at values of $x=0,0.2,0.4,0.5,0.6,0.8$ and 1 . We examine whether the Se : Te composition of the QDs can be tuned, while keeping other parameters (QD size) constant.

XRD characterization. Fig. 4 shows the XRD patterns of these samples series. As for the previous series, the XRD patterns of the alloyed CdTeSe QDs show peaks corresponding to (111), (220) and (311) planes of cubic zinc-blende phase of CdTeSe. The position of the three diffraction lines are in between the 


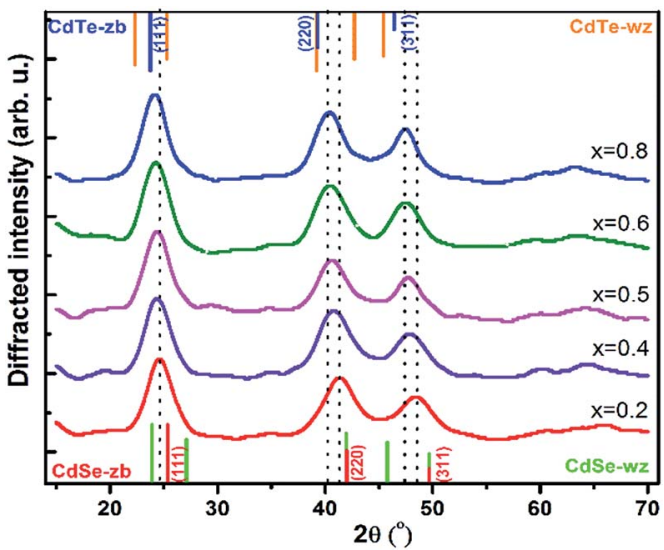

Fig. 4 Powder XRD patterns of CdTeSe ternary QDs prepared at temperature $260^{\circ} \mathrm{C}$ (for Te/(Se $+\mathrm{Te}$ ) proportions $x=0.2,0.4,0.5,0.6$ and 0.8 ). The tabulated values of the bulk diffraction peaks for zinc blend (zb) and wurtzite (w) CdTe, CdSe are shown.

lines of the two crystalline phases zb-CdSe and zb-CdTe. The position of these peaks were all shifted towards lower angles $2 \theta$ when $x$ changed from 0.2 to 0.8 . For $x=0.2$, the peaks are near the tabulated CdSe lines, while for $x=0.8$ the peaks are near the CdTe tabulated values. This indicates that the alloy composition of the synthesized QDs is tuned as a result of the change in Te/ Se reagent ratio, starting from Se-rich QDs to Te-rich QDs.

Raman spectra. Fig. 5 shows the Raman scattering spectra of this CdTeSe QD sample series, along with the spectra for pure CdSe and CdTe QDs. When $x=0.2$, the alloy ternary sample contains mostly Se: on the Raman spectra only one peak appears corresponding to the CdSe-like LO phonon, at the frequency $200 \mathrm{~cm}^{-1}$, as reported for CdSe in ref. 39 and found also in for our CdSe QDs. From $x=0.4$ to 0.8 , a second peak appears and gradually increases at $159 \mathrm{~cm}^{-1}$ characteristic for the CdTe-like LO phonon of ternary alloy QDs, showing the increase of Te concentration. One significant thing is that when $x=0.5$ or higher, the CdSe peak is shifted to $188 \mathrm{~cm}^{-1}$, similar to the Raman spectra of CdTeSe ternary alloy QDs presented on

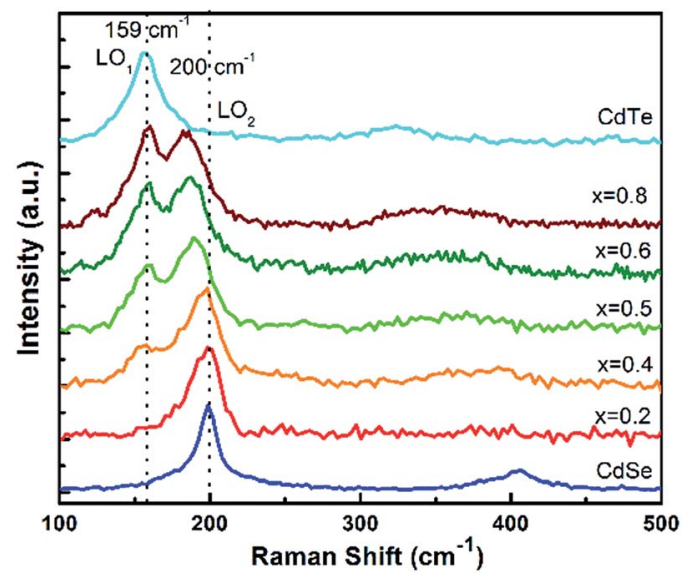

Fig. 5 Raman spectra of $\mathrm{CdTe}_{x} \mathrm{Se}_{1-x}$ QDs prepared at temperature $260^{\circ} \mathrm{C}, x=0.2,0.4,0.5,0.6$ and 0.8 , along with pure CdSe and CdTe QDs.

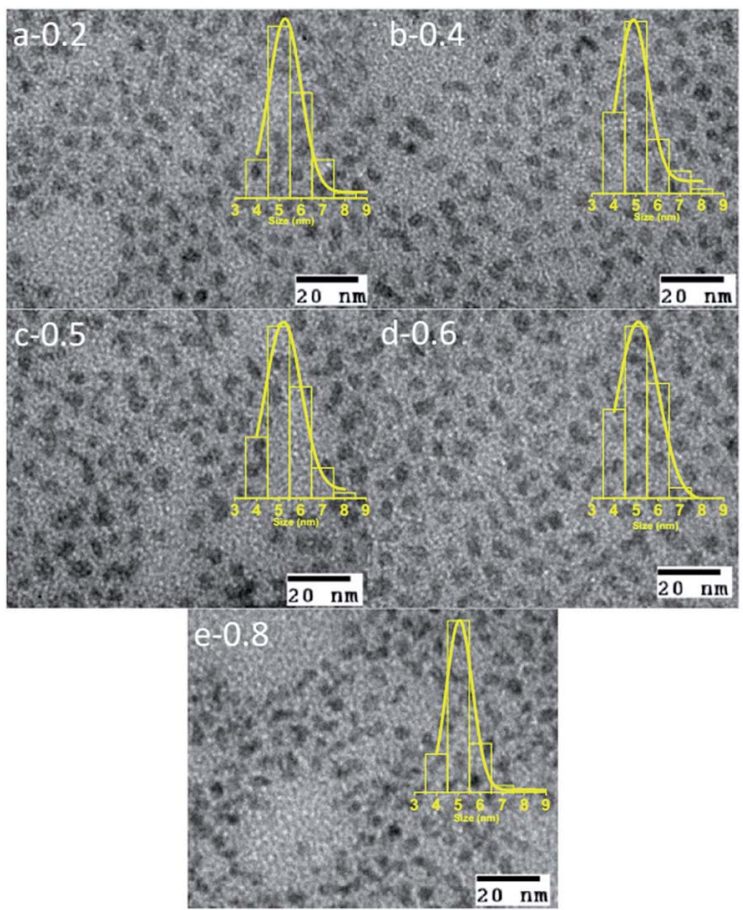

Fig. 6 TEM images of the $\mathrm{CdTe}_{x} \mathrm{Se}_{1-x}$ QDs samples prepared at the temperature $260^{\circ} \mathrm{C}(10 \mathrm{~min}), x$ varies from 0.2 to 0.8 corresponding to images (a-e). Scale bars: $20 \mathrm{~nm}$.

Fig. 3. This shift can probably not be explained by a change in crystalline structure as it is known to remain zinc-blende from XRD data. However, the mixing of CdTe and CdSe can lead to a lattice parameter different from the bulk one, so that the phonon frequency is modified depending on the alloy composition (a similar effect was reported in $^{38}$ for CdSeS, with a strong shift of the CdS phonon - and much lower shift of the CdSe phonon).

TEM images. Fig. 6 shows the TEM images of the different samples. The particles have a rather cubic shape and a rather uniform average size of $\sim 5.4-5.1 \mathrm{~nm}$ that barely changes when $x$ increases from 0.2 to 0.8 . Therefore, with our growth protocol,

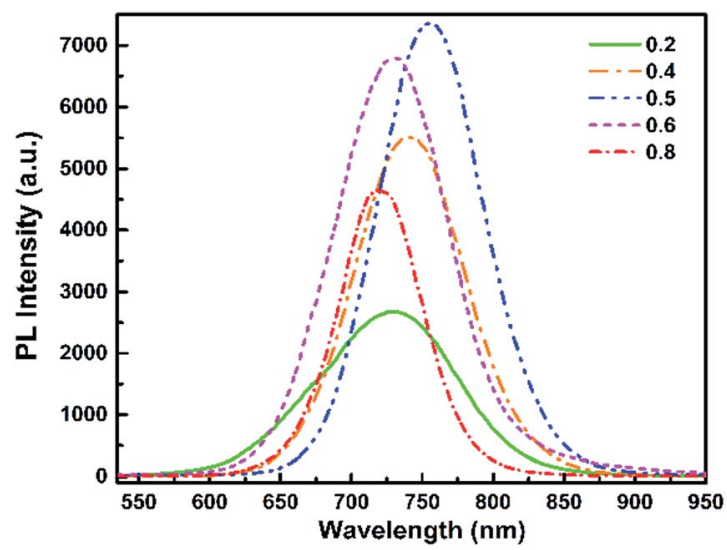

Fig. 7 The emission spectra of $\mathrm{CdTe}_{x} \mathrm{Se}_{1-x}$ QD samples $(x=0.2,0.4$, $0.5,0.6$ and 0.8$)$ grown at temperature $260{ }^{\circ} \mathrm{C}, \lambda_{\text {exc. }}=532 \mathrm{~nm}$. 


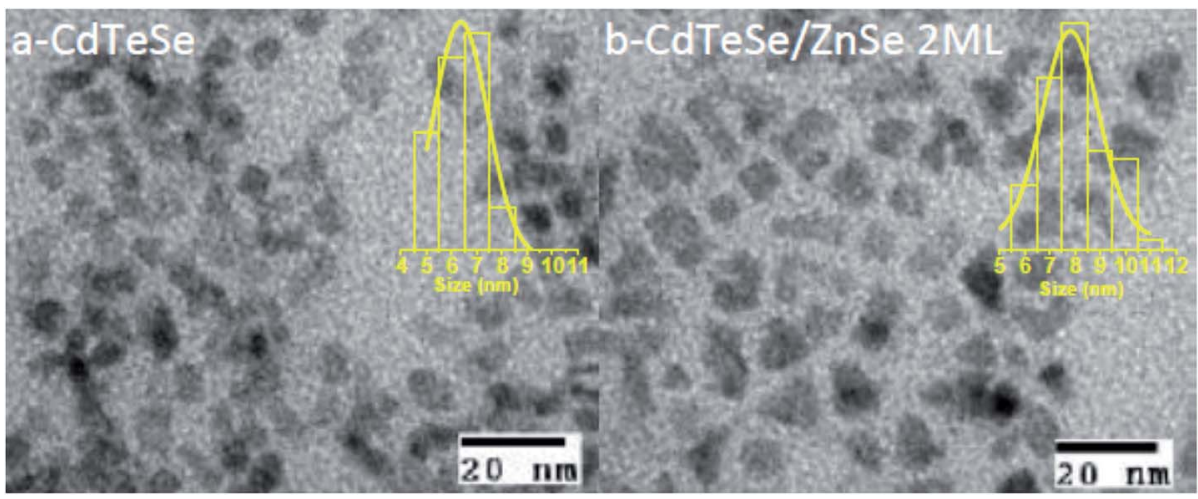

Fig. 8 TEM images of the CdTeSe QDs sample prepared at temperature $260^{\circ} \mathrm{C}$ (a) and CdTeSe/ZnSe $2 \mathrm{ML}$ QDs(b), size: (a) $=6.3 \mathrm{~nm}$, (b) $=$ $8.3 \mathrm{~nm}$. Scale bars: $20 \mathrm{~nm}$.

the composition can be tuned from Se-rich to Te-rich while keeping the QD size constant.

Photoluminescence properties. Fig. 7 shows the photoluminescence spectra of the samples. As the amount of Te reagent is increased, the PL is redshifted from $680 \mathrm{~nm}(x=$ $0.2)$ to $750 \mathrm{~nm}(x=0.5)$ and then blueshifted to $715-720 \mathrm{~nm}$ $(x=0.6-0.8)$. This non-monotonic behavior is most likely caused by the optical bowing effect: the bandgap of an alloy is not a monotonic function of its composition. The PL intensity is larger for the $x=0.5-0.6$ samples and decreases for smaller or larger ratios $x$, while remaining though much larger than in Fig. 1 due to the removal of OLA from the precursors solution.

\section{Study on the alloyed CdTeSe/ZnSe QDs}

We finally consider a series of CdTeSe samples fabricated at $260{ }^{\circ} \mathrm{C}$ with $x=0.5$ and shelled with a ZnSe layer of different thicknesses. Two samples were considered for more detailed studies: CdTeSe cores and CdTeSe shelled with ZnSe 2 ML nominal thickness.

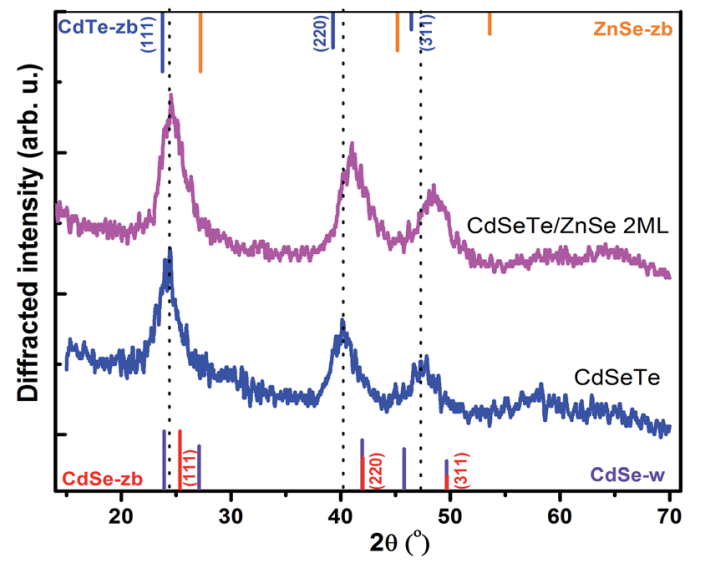

Fig. 9 Powder XRD patterns of CdTeSe ternary QDs and CdTeSe/ ZnSe $2 \mathrm{ML}$ prepared at temperature $260^{\circ} \mathrm{C}$. The tabulated values of the bulk diffraction peaks for zinc blend (zb) CdTe, (zb) CdSe, (w) CdSe and $(\mathrm{zb}) \mathrm{ZnSe}$ are shown.
TEM images. TEM images of these two samples are presented on Fig. 8. It can be seen that the nanocrystals in the samples have a strongly facetted shape. Generally, none of them is spherical. The average size (long axis) among them is $6.3 \mathrm{~nm}$ for the CdTeSe core QDs and $8.3 \mathrm{~nm}$ for the QDs shelled with ZnSe 2 ML. A similar facetted shape has also been observed and reported in ref. 4.

XRD characterization. Fig. 9 shows their XRD patterns, on which we can see again that the CdTeSe QDs crystallize at the zb-CdTeSe phase. On the XRD pattern of the sample shelled with ZnSe $2 \mathrm{ML}$, the presence of only three diffraction lines also indicate a zb-phase, with epitaxial growth of the shell. The positions of these lines are shifted towards slightly higher angles $2 \theta$ due to the addition of the ZnSe shell (although it could be related also to an incorporation of Se inside the core during the shell growth, as both $\mathrm{ZnSe}$ and CdSe lines are tabulated at higher diffraction angles).

Raman spectra. The Raman scattering spectra of these samples are similar to those published in ref. 20 as presented on Fig. 10. On the Raman spectra of the shelled samples appears a vibration line with high intensity, characteristic for the LO mode of CdSe at $200 \mathrm{~cm}^{-1}$ (ref. 40) and a shoulder with

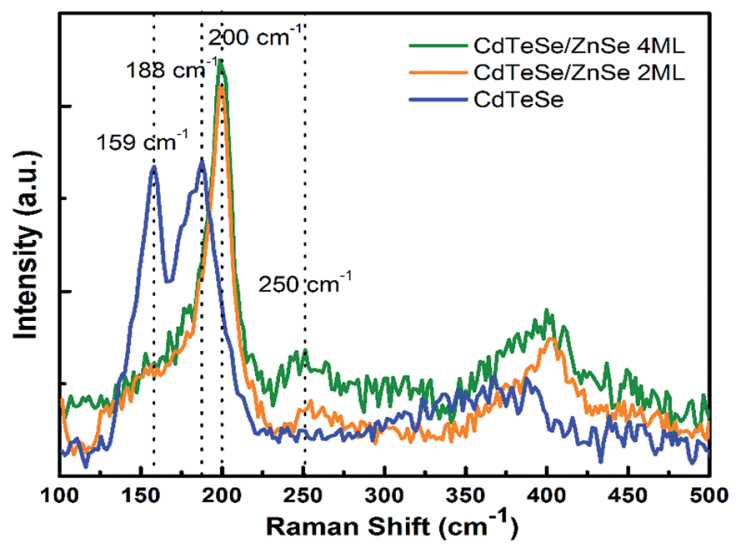

Fig. 10 Raman spectra of alloyed CdTeSe QDs cores and cores coated with shells of ZnSe with different monolayer thicknesses ( $\mathrm{MML}$, $\mathrm{n}=2$ and 4 ). 

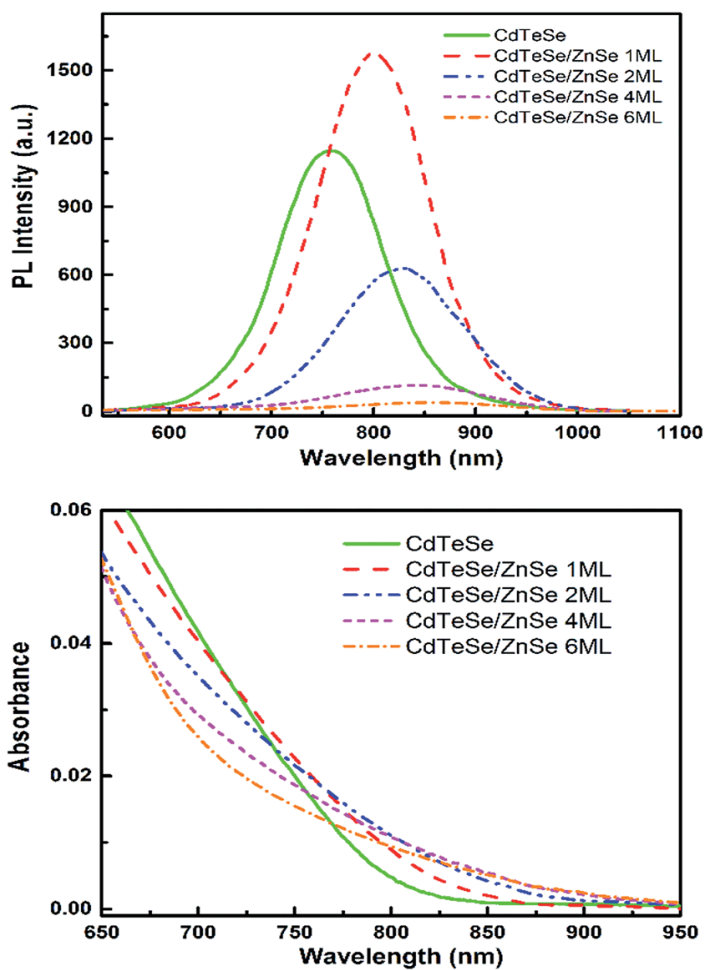

Fig. 11 Photoluminescence (top) and absorption (bottom) spectra of the CdTeSe and CdTeSe coated with shells of ZnSe with different monolayer thicknesses ( $n M L, n=1,2,4$ and 6).

low intensity at $159 \mathrm{~cm}^{-1}$. This peak does not change as the shell thickness increases. This result suggests that, when the $\mathrm{Zn}$ and Se precursors are introduced for the shell growth, excess $\mathrm{Cd}$ ions might still be present, so that a CdSe material layer forms gradually on the CdTeSe core, thus we obtain CdSe-rich QDs. On the other hand, the ZnSe layer still competes to form along side CdSe. This can be seen on the Raman spectra with the phonon band characteristic for ZnSe at $\sim 250 \mathrm{~cm}^{-1}$, despite its weak intensity. The results on the Raman spectra show that the ZnSe shell has been formed, but in a small amount. Therefore, a layer of CdSe-rich CdTeSe shelled the CdTeSe cores, and on the Raman spectra we can observe characteristic lines for CdSe-rich QDs as well as the vibration line characteristic for ZnSe at $\sim 250 \mathrm{~cm}^{-1}$ but with weak intensity.

Ensemble optical properties. Fig. 11 shows the absorption spectra and normalized photoluminescence (PL) spectra of all samples of alloyed CdSeTe core QDs and coated with the ZnSe shells up to 6 ML nominal thickness. The PL spectrum of the CdTeSe core sample is a wide emission band whose peak position is located at the wavelength $760 \mathrm{~nm}$. When coated with a layer of ZnSe, the emission band peak position is shifted towards longer wavelengths, up to $842 \mathrm{~nm}$ when coated with ZnSe $2 \mathrm{ML}$, and up to $882 \mathrm{~nm}$ when coated with ZnSe $6 \mathrm{ML}$. The full width at half maximum (FWHM) of the emission band of CdTeSe core is $126 \mathrm{~nm}$, which increases when coated with more layers of ZnSe. The PL decay curves (Fig. SI-3†) show an increase of their fast component upon shell growth. This might indicate the presence of defects in the shell, as well as a penetration of the charges into the shell, which would be in agreement with a thin layer of ZnSe. The PL efficiency is increased for the thinner $1 \mathrm{ML}$ shell, but begins to decreased for the $2 \mathrm{ML}$ sample (although their single-QD properties remain good as will be shown below) and drops sharply for the $4 \mathrm{ML}$ and $6 \mathrm{ML}$ sample, again indicating the presence of defects for the thickest shells.

The absorption edge of CdTeSe QD cores is located at $820 \mathrm{~nm}$ wavelength, and is shifted towards longer wavelengths - by $50 \mathrm{~nm}$ when coated with a ZnSe layer 2 ML thick, and $8 \mathrm{~nm}$ when coated a layer ZnSe layer 6 ML thick. The absorption band expanding up to $\sim 900 \mathrm{~nm}$ is an interesting feature for applications in solar cells. This redshift might be related to a penetration of the charges into an additional CdSe layer, although CdSe has a larger band gap; an increase of the Se concentration in the CdTeSe alloy would also lead to a blueshift, as shown in Fig. 9. A possible explanation for the large redshift could be the appearance of a type-II structure in these QDs, which would also explain the relatively long radiative decay time observed on single QDs (see Fig. 13 below). Further analysis, for instance
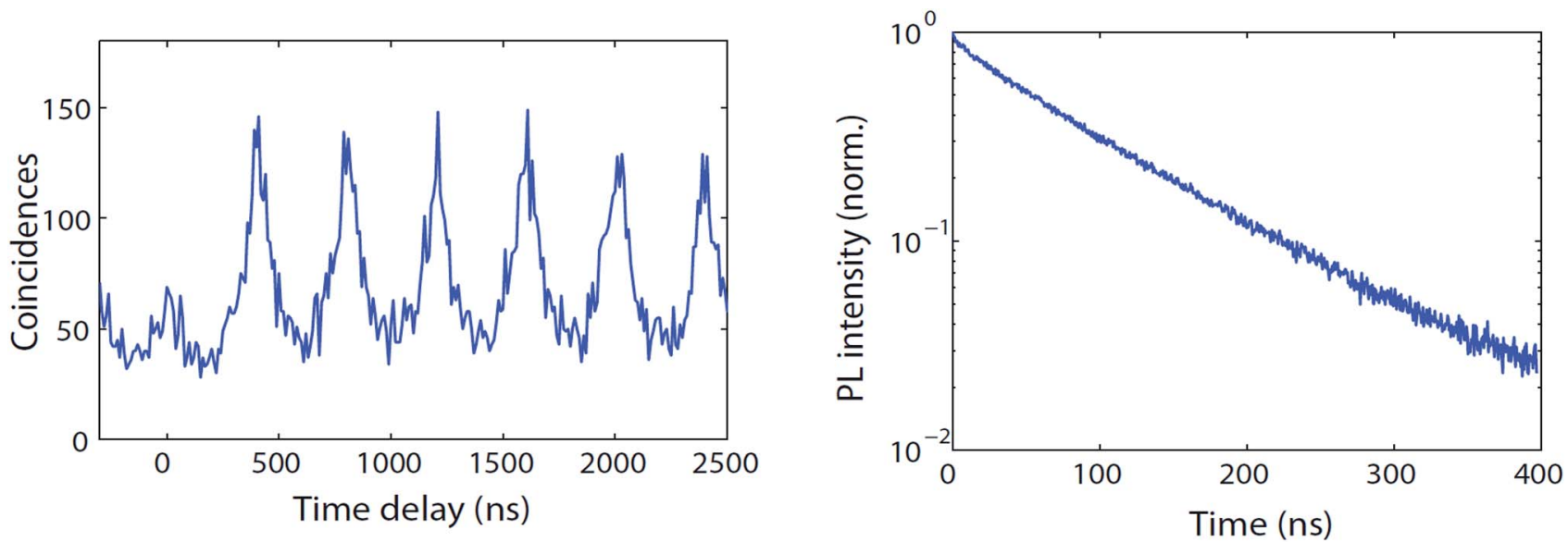

Fig. 12 Left: PL intensity autocorrelation $g^{(2)}$ function (arb. units) of a typical individual CdTeSe quantum dot. Right: Decay curve (norm.) of the same quantum dot. 


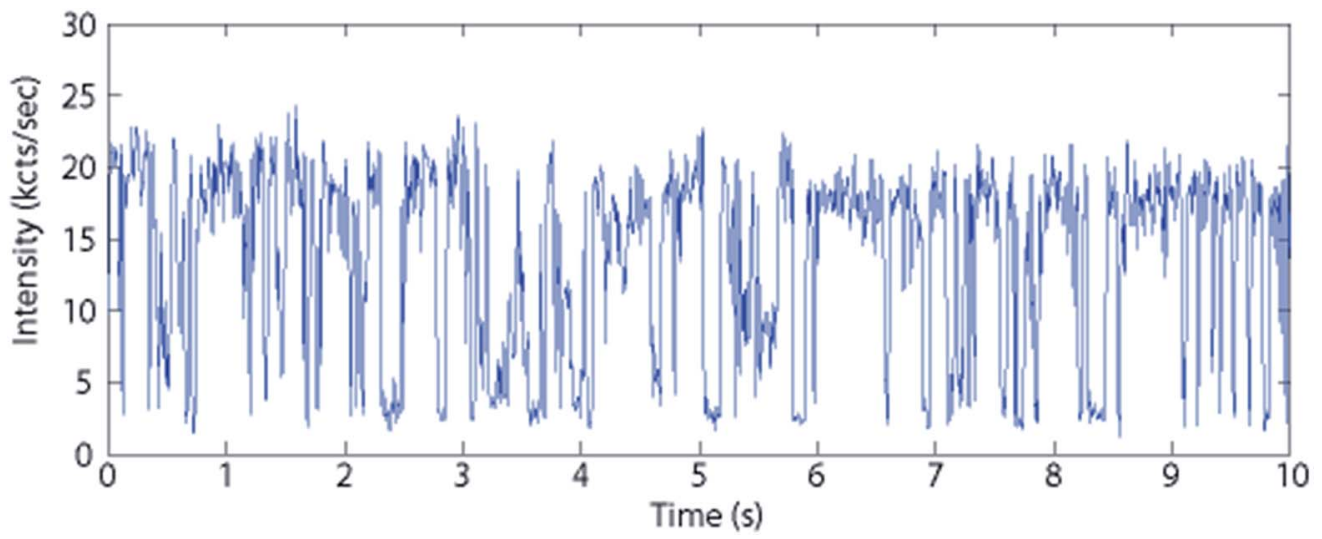

Fig. 13 Intensity-time trace of a typical CdTeSe/ZnSe quantum dot.

HR-TEM imaging of the QDs, would be necessary in order to elucidate this aspect, which is important as NIR absorption and slow radiative decay are key elements for photovoltaic applications.

\section{Single-QD optical properties}

We now use a microphotoluminescence setup to analyze the luminescence of individual CdTeSe/ZnSe quantum dots. Fig. 12 (left) shows the PL intensity autocorrelation curve (distribution of delays between one photon and the next photon detected) of a typical emitter under excitation by a pulsed laser with $400 \mathrm{~ns}$ pulse repetition period. The peaks at times multiples of $400 \mathrm{~ns}$ indicate that all photons are emitted slightly after a laser pulse so that the delay between two photons is roughly a multiple of 400 ns. The nearly-perfect absence of a peak at zero delay indicates that there is never emission of two photons following the same laser pulse. This indicates that for these CdTeSe/ZnSe nanocrystals we have obtained single-photon emission, most likely because multi-exciton emission is quenched by Auger effect. The minor residual peak might be due to selfluminescence from the substrate, possibly with a slight contribution from multi-exciton emission.

Fig. 12 (right) plots the decay curve of the same quantum dot. This curve is remarkably close to a mono-exponential, with an unusually long decay time of $110 \mathrm{~ns}$. This observation, which was reproduced for all single quantum dots observed with similar $110 \pm 15 \mathrm{~ns}$ decay times, is in contrast with ensemble measurements, possibly because the latter are performed at much higher power which could excite multi-excitonic or other non-radiative recombination pathways (the increase of multiexcitonic contribution upon shell growth could then be explained by decreased competition with Auger decay). Under the single-QD observation conditions, there is no (fast) multiexcitonic contribution and, during the measurement (100 seconds), there were very few fluctuations of the decay time. This excellent stability is confirmed by considering the intensity variations of a typical quantum dot (Fig. 13). Some nonfluorescent periods ("off") are observed, but they constitute only $20 \%$ of the total time (and less than $10 \%$ for many other
QDs). During the "on" periods, the emission remains remarkably stable.

\section{Conclusions}

In summary, CdTeSe QDs with up to $860 \mathrm{~nm}$ emission bands have been fabricated. The absorption intensity of these QDs starts to become stronger at wavelengths $\sim 850 \mathrm{~nm}$ when fabricated at $260{ }^{\circ} \mathrm{C}$. The QDs crystallize stably at CdTeSe zincblende phase at the growth temperature of $260{ }^{\circ} \mathrm{C}$. The NIR absorbing properties at NIR make CdTeSe QDs usable in solar cells. Raman scattering spectra can be used as a powerful tool to evaluate qualitatively the amount of Te:Se in the alloy CdTeSe QDs. When the nanocrystal growth temperature is increased, their size increases as well as the amount of Se in the QDs. When the amount of Te reagent is increased, the intensity of the CdTe-like LO line increases compared to that of the CdSe-like LO line in CdTeSe QDs, showing increased Te concentration in the QD - the QD size, on the other hand, can be kept constant. Upon growth of a ZnSe shell, the emission band is redshifted up to $900 \mathrm{~nm}$. For single core-shell nanocrystals, perfect single-photon emission is obtained, with good stability and little intermittency.

\section{Conflicts of interest}

There are no conflicts to declare.

\section{Acknowledgements}

This research is funded by Vietnam National Foundation for Science and Technology Development (NAFOSTED) under grant number 103.03-2014.66 and by the CNRS-VAST programm Plato (PICS 6456). P. Bassène is supported by the Service de Coopération et d'Action Culturelle of the French Embassy in Dakar. Fu Feng is supported by the ANR-12-JS04-0011 project Ponimi. The authors thank the National Key Laboratory for Electronic Materials and Devices - IMS and Duy Tan University for the use of facilities. We would like to thank Mr Pham Son Lam for his 
assistance in editing the paper and Prof. Vu Xuan Quang of Duy Tan University for his support.

\section{Notes and references}

1 R. Wang, Y. Shang, P. Kanjanaboos, W. Zhou, Z. Ning and E. H. Sargent, Energy Environ. Sci., 2016, 9, 1130-1143.

2 W. W. Yu, L. Qu, W. Guo and X. Peng, Chem. Mater., 2003, 15, 2854-2860.

3 L. Li, Y. Chen, Q. Lu, J. Ji, Y. Shen, M. Xu, R. Fei, G. Yang, K. Zhang, J.-R. Zhang and J.-J. Zhu, Sci. Rep., 2013, 3, 01529.

4 R. E. Bailey and S. Nie, J. Am. Chem. Soc., 2003, 125, 71007106.

5 J. Xue, X. Chen, S. Liu, F. Zheng, L. He, L. Li and J. J. Zhu, ACS Appl. Mater. Interfaces, 2015, 7, 19126-19133.

6 H. Zou, M. Liu, D. Zhou, X. Zhang, Y. Liu, B. Yang and H. Zhang, J. Phys. Chem. C, 2017, 121, 5313-5323.

7 J. Yang, J. Wang, K. Zhao, T. Izuishi, Y. Li, Q. Shen and X. Zhong, J. Phys. Chem. C, 2015, 119, 28800-28808.

8 Z. Du, Z. Pan, F. Fabregat-Santiago, K. Zhao, D. Long, H. Zhang, Y. Zhao, X. Zhong, J. S. Yu and J. Bisquert, J. Phys. Chem. Lett., 2016, 7, 3103-3111.

9 S. K. Verma, R. Verma, N. Li, D. Xiong, S. Tian, W. Xiang, Z. Zhang, Y. Xie and X. Zhao, Sol. Energy Mater. Sol. Cells, 2016, 157, 161-170.

10 G. Wang, H. Wei, Y. Luo, H. Wu, D. Li, X. Zhong and Q. Meng, J. Power Sources, 2016, 302, 266-273.

11 R. Soltania, A. A. Katbaba, K. Schaumbergerb, N. Gasparinib, C. J. Brabec, S. Rechbergerd, E. Spieckerd, A. G. Alabaue, A. Rulandf, A. Sahag, D. M. Guldig, V. Sgobba and T. Ameri, J. Mater. Chem. C, 2017, 5, 654-662.

12 X. Zhong, M. Han, Z. Dong, T. J. White and W. Knoll, J. Am. Chem. Soc., 2003, 125, 8589-8594.

13 Z. Pan, K. Zhao, J. Wang, H. Zhang, Y. Feng and X. Zhong, ACS Nano, 2013, 7(6), 5215-5222.

14 W. Jiang, A. Singhal, J. Zheng, C. Wang and W. C. W. Chan, Chem. Mater., 2006, 18, 4845-4854.

15 T. Pons, N. Lequeu, B. Mahler, S. Sasnousk, A. Fragola and B. Dubertret, Chem. Mater., 2009, 21, 1418-1424.

16 B. Xing, W. Li, X. Wang, H. Dou, L. Wang, K. Sun, X. He, J. Han, H. Xiao, J. Miao and Y. Li, J. Mater. Chem., 2010, 20, 5664.

17 R. Wang, O. Calvignanello, C. I. Ratcliffe, X. Wu, D. M. Leek, M. B. Zaman, D. Kingston, J. A. Ripmeester and K. Yu, J. Phys. Chem. C, 2009, 113, 3402-3408.

18 Z. Wan, W. Luan and S. T. Tu, J. Colloid Interface Sci., 2011, 356, 78-85.

19 L. Song, J. Duan and J. Zhan, Mater. Lett., 2010, 64, 18431845.
20 L. X. Hung, P. N. Thang, H. Van Nong, N. H. Yen, V. Đ. Chinh, L. Van Vu, N. T. T. Hien, W. D. de Marcillac, P. N. Hong, N. T. Loan, C. Schwob, A. Maître, N. Q. Liem, P. Bénalloul, L. Coolen and P. T. Nga, J. Electron. Mater., 2016, 45, 4425-4431.

21 T. Debnath, S. Maiti and H. N. Ghosh, J. Phys. Chem. Lett., 2016, 7, 1359-1367.

22 L. Liu, X. Xu, T. Luo, Y. Liu, Z. Yang and J. Lei, Solid State Commun., 2012, 152, 1103-1107.

23 L. Liao, H. Zhang and X. Zhong, J. Lumin., 2011, 131, 322327.

24 F. Yang, Z. Xu, J. Wang, F. Zan, C. Dong and J. Ren, Luminescence, 2013, 28, 392-400.

25 H. Y. Nguyen, W. D. de Marcillac, C. Lethiec, N. H. Phan, C. Schwob, A. Maître, Q. L. Nguyen, V. V. Le, P. Bénalloul, L. Coolen and P. Thu Nga, Opt. Mater., 2014, 36, 1534-1541.

26 J. D. Poplawsky, W. Guo, N. Paudel, A. Ng, K. More, D. Leonard and Y. Yan, Nat. Commun., 2016, 7, 12537.

27 V. Dzhagan, I. Lokteva, C. Himcinschi, X. Jin, J. KolnyOlesiak and D. R. Zahn, Nanoscale Res. Lett., 2011, 6, 79.

28 S. Li, G. Tan, J. B. Murowchick, C. Wisner, N. Leventis, T. Xia, X. Chen and Z. Peng, J. Electron. Mater., 2013, 42, 3373-3378.

29 B. T. Spann and X. Xu, Appl. Phys. Lett., 2014, 105, 083111.

30 Z. Chai, W. Wu, D. Kong, Y. Gao and Q. Chang, J. Non-Cryst. Solids, 2013, 382, 121-124.

31 D. N. Talwar, Z. C. Feng, J.-F. Lee and P. Becla, Phys. Rev. B: Condens. Matter Mater. Phys., 2013, 87, 1652018.

32 E. S. F. Neto, S. W. da Silva, P. C. Morais, M. I. Vasilevskiy, M. A. Pereira-da-Silva and N. O. Dantas, J. Raman Spectrosc., 2011, 42, 1660-1669.

33 S. Perkowitz, L. S. Kim and P. Becla, Phys. Rev. B: Condens. Matter Mater. Phys., 1991, 43, 6598-6601.

34 D. N. Talwar, T.-R. Yang, Z. C. Feng and P. Becla, Phys. Rev. B: Condens. Matter Mater. Phys., 2011, 84, 174203.

35 H. W. Verleur and J. A. S. Barker, Phys. Rev., 1966, 149, 715729.

36 V. S. Vinogradov, G. Karczewski, I. V. Kucherenko, N. N. Mel'nik and P. Fernandez, Phys. Solid State, 2011, 50, 164-167.

37 A. V. Bragas, C. Aku-Leh and R. Merlin, Phys. Rev. B: Condens. Matter Mater. Phys., 2006, 73, 125305.

38 P. Mukherjee, S. J. Lim, T. P. Wrobel, R. Bhargava and A. M. Smith, J. Am. Chem. Soc., 2016, 138, 10887-10896.

39 V. M. Dzhagan, M. Y. Valakh, A. E. Raevskaya, A. L. Stroyuk, S. Y. Kuchmiy and D. R. T. Zahn, Nanotechnology, 2007, 18, 285701.

40 V. M. Dzhagan, M. Y. Valakh, O. E. Raevska, O. L. Stroyuk, S. Y. Kuchmiy and D. R. Zahn, Nanotechnology, 2009, 20, 365704 . 